\title{
一种有效的多室结构微胶囊制备方法: 多流体复合电喷技术
}

陈洪燕, 赵勇", 江雷"

中国科学院化学研究所, 北京 100190

* 联系人, E-mail: zhaoyong@iccas.ac.cn; jianglei@iccas.ac.cn

2008-11-29 收稿, 2009-02-26 接受

国家自然科学基金(批准号: 20801057, 20774101). 国家重点基础研究发展计划(编号: 2007CB936403, 2009CB930404)和国家高技术研究发 展计划(编号: 2007AA03Z327)资助项目

摘要 具有多室结构的微胶囊在智能药物输运、微反应器、复杂传感器等方面具有重要的应用 前景. 提出了一种新颖的多流体复合电喷技术, 可一步实现多室结构微胶囊的制备. 该胶囊内 部各室间有内壁相隔, 每一个内室可独立的封装不同组分, 各组分间互不接触. 将一定数目的 较细的内管插于较粗的外管组装了具有分级结构的复合喷头, 这种独特的结构可以使电喷过程 中壳材料形成对核材料的有效并且独立的包覆, 从而导致了多组分同时包覆的多室结构微胶囊 的制备. 通过对复合喷头结构的设计可实现对腔室的数目以及相应的封装组分的数目的控制, 并且可方便地扩展到其他功能材料的制备. 该法制备的微胶囊不仅可以扩展单一胶囊的功能, 而且其独特的多室结构可对被封装的核材料进行保护, 尤其适用于活性材料如环境敏感性材料 及反应性材料的同时包覆, 将在多组分药物输运、微反应器等领域具有潜在的应用前景.

关键词

微胶囊

电喷雾

多室结构

多组分

微纳米材料
微胶囊技术在生物医药、食品科学、材料学等领 域都有着广泛应用. 目前人们已经发展了很多优异 的物理和化学的微胶囊制备方法, 如微流体技术、自 组装技术、乳化法、同轴电喷法等 ${ }^{[1 ~ 10]}$. 近年来, 由 于在生物医药领域的应用前景, 具有多室结构的微 胶囊越来越引起人们的关注. 多室结构微胶囊可以 将多种敏感的生物材料如酶、蛋白质、核酸、疫苗等 集成到同一个微粒实体当中并对它们进行分隔保护. 这样不仅可以靶向输运这些功能组分, 而且可以选 择性的释放单一组分或混合组分. 这种独特的结构 不仅可以扩大单一胶囊的功能，而且可通过外场刺 激来控制包覆组分的分离和混合从而引发反应, 因 而在智能药物输运、微反应器、复杂传感器等方面具 有重要的应用前景.

目前报道的多室结构微胶囊的制备方法主要为
自组装法以及乳化法等. 聚电解质层层自组装法 (layer-by-layer self-assembly)是制备多室结构微胶囊 的一种有效方法. 德国马普所的Kreft等利用该方法 制备了具有同心双室结构的“壳套壳”(shell-in- shell) 结构微胶囊 $[11]$. 他们以碳酸钲为牺牲模板首先制备 了负载有不同生物大分子的同心“球套球” 结构粒子, 通过在每层碳酸钙的表面自组装引入聚电解质层并 除去模板, 最终获得以聚电解质层为壁材料的多室 结构微胶囊. 这种结构的微胶囊内部可以独立的封 装两种不同的生物大分子, 并且由于聚电解质壁层 具有半渗透性, 小分子可以自由通过而大分子被阻 隔, 因而可以用作生物反应的微反应器. 进一步将光 吸收的金纳米粒子引入到内层壳壁上, 他们实现了 用近红外激光辐射来破坏内层隔壁, 从而远程控制 不同室间成分的混合与反应 ${ }^{[12]}$. 在小分子的自组方 
面, Vogel等利用磷脂分子自组装构建了一种大磷脂 囊泡包裹多个小磷脂囊泡的多反应室集成微反应器, 利用磷脂囊泡的渗透性在特征有序流体相转变温度 左右会改变的特性，通过远程控温即可实现小磷脂 囊泡内部包覆物质的释放及其与大囊泡内负载材料 的反应 ${ }^{[13]}$.

两亲性嵌段共聚物在水溶液中的自组装由于在 缓释、纳米材料的制备、分散等方面的应用而引起了 广泛的研究兴趣. 与 $A B$ 两嵌段和 $A B A$ 三嵌段共聚物 相比, $\mathrm{ABC}$ 三嵌段共聚物在水溶液中的自组装行为 更为复杂. 通过改变 $A, B, C$ 各链段间的相容性以及 共聚物的结构, 可实现多室结构胶束的制备. Jérôme 等报道了聚苯乙烯-b-聚(2-乙烯基嘧啶)-b-聚氧乙烯 三嵌段共聚物在水溶液中可自组装形成核/壳/冠三层 结构胶束, 并且由于壳层的聚(2-乙烯基嘧啶)的水溶 解性具有 $\mathrm{pH}$ 响应，因而整个胶束结构具有 $\mathrm{pH}$ 响应性 [14]. Hillmyer等报道聚氧乙烯-b-聚苯乙烯-b-1, 2-聚丁 二烯 $(\mathrm{OSB})$ 三嵌段共聚物在水溶液中可形成聚氧乙烯 为壳层, 另外两疏水嵌段共混为核层的球形结构胶 束. 进一步选择性的将聚丁二烯链段氟化增大链段 间的不相容性, 该共聚物可自组装成为核/壳/冠三层 结构胶束 ${ }^{[15]}$. 另外, 他们将三种相互之间不混溶的聚 合物(可溶于水的聚氧乙烯, 饱和碳水化合物聚乙基 乙烯, 疏水疏油的聚全氟氧丙稀)共聚形成三臂星形 $\mathrm{ABC}$ 三嵌段共聚物, 由于链段的相容性差异及其特 殊的三重连接点结构该共聚物在稀水溶液中可以自 组装形成不同于传统核/壳/冠层结构的多室结构胶束, 并且通过调节聚合物链段的长度可以控制自组装结 构由离散的多室结构胶束逐渐转变为具有多个分离 核的蠕虫状长胶束 ${ }^{[16]}$. Thünemann等人合成了具有对 称结构的线型 $\mathrm{ABCBA}$ 五嵌段共聚物( $\mathrm{A}$, 聚氧乙烯; B, 聚( $(\gamma$-芐基- $-\mathrm{L}$-谷胺酸酯); $\mathrm{C}$, 聚全氟乙醚). 由于 3 种聚 合物构筑单元的相容性差异很大, 因而在水溶液中 自组装主要形成长度为 $100 \sim 200 \mathrm{~nm}$, 直径约为 $24 \mathrm{~nm}$ 的柱状两室结构胶束 ${ }^{[17]}$.

乳化法是制备微胶囊的一种有效方法. 近年来 随着微流体技术的兴起, 人们发现这项技术是可用 来可控制备多乳液, 这些多乳液经进一步处理可获 得各种结构的微胶囊. Nisisako等用十字交叉疏水性 上游节点和 $\mathrm{T}$ 型亲水性下游节点的微流体通道结构设 计实现了两种不同水滴同时包覆于一个油滴的双组 分 $\mathrm{W} / \mathrm{O} / \mathrm{W}$ 双乳液的制备. 这种方法表明多种组分可
以独立的封装到皮升/纳升级的液滴当中，因而在生 物芯片等领域具有潜在的应用前景 ${ }^{[1]}$. Chu等报道了 利用毛细管微流体装置可制备单分散、内部液滴数目 及尺寸可控的多乳液. 他们进一步由三乳液制备了 温度响应水凝胶微胶囊, 通过调节温度可控制内部 包覆两相(油相和水相)物质的释放 ${ }^{[187]}$.

但是上述多室结构微胶囊的制备方法各具有一 定的局限性, 如自组装技术, 它是一个多步过程, 即 一次操作一般只能封装一种物质(一室的制备), 当需 要封装多种组分尤其是活性组分时, 为了保护各组 分的活性，难免会导致操作繁琐、封装效率低下等问 题. 而且大量界面的存在也大大增加了组分失活的 几率. 因而寻找一种简便高效且可控的多室结构微 胶囊制备方法具有十分重要的意义. 美国密歇根大 学的Roh等人报道了一种“肩并肩”电喷的方法, 可以 将两种或 3 种不同的材料结合到同一个粒子当中, 制备出具有“双面神”或三相结构的各向异性微纳米 粒子 ${ }^{[19,20]}$. 虽然该方法制备的粒子不具有微胶囊结 构, 但为如何实现多种组分的可控封装提供了新的 思路.

我们提出了一种新颖的多流体复合电喷技术, 可一步实现多室结构微胶囊的制备. 该胶囊内部各 室间有内壁相隔, 可分别独立的封装不同的组分, 并 且腔室的数目以及相应的封装组分的数目可方便的 进行控制. 我们制备了有机物、有机/无机复合两种多 室微胶囊, 对其进行了扫描电子显微镜、透射电子显 微镜、光学显微镜以及电流电压性质进行了表征, 并 拍摄了复合电喷过程中形成的 Taylor锥照片, 初步解 释了多室结构形成的机理. 结果表明, 该多流体复合 电喷技术是制备多室结构微胶囊的有效方法, 在多 组分药物输运、微反应器等领域具有潜在的应用前景 [21].

\section{1 材料和方法}

Somos 14120 (美国 DSM 公司), 聚乙烯基吡咯烷 酮 K30 (PVP)(北京益利精细化学品有限责任公司), 铁酸四正丁酯 $\left[\mathrm{Ti}(\mathrm{OBu})_{4}\right]$ (北京精细化学品有限责任 公司), 无水乙醇(分析纯, 北京精细化学品有限责任 公司), 冰醋酸(分析纯, 北京精细化学品有限责任公 司), 液体石蜡(汕头西陇化工厂有限公司), 丙三醇 (分析纯, 国药集团化学试剂有限公司). 红、黄、蓝 色染料由中国科学院理化技术研究所友情提供, 结 
构式如下:<smiles>CC1=NN(c2ccc(S(=O)(=O)O)cc2)C(=O)/C1=C\C=C/C=C\c1c(C)nn(-c2ccc(S(=O)(=O)O)cc2)c1O</smiles><smiles>CC1=NN(c2ccc(S(=O)(=O)O)cc2)C(=O)/C1=C/C=C\c1c(C)nn(-c2ccc(S(=O)(=O)O)cc2)c1O</smiles><smiles>CC1=NN(c2ccc(S(=O)(=O)O)cc2)C(=O)/C1=C\c1c(C)nn(-c2ccc(S(=O)(=O)O)cc2)c1O</smiles>

表 1 流体性质参数汇总

\begin{tabular}{lccc}
\hline \multicolumn{1}{c}{ 材料 } & 黍度 $/ \mathrm{mPa} \cdot \mathrm{s}$ & $\begin{array}{c}\text { 表面张力/ } \\
\mathrm{mN} \cdot \mathrm{m}^{-1}\end{array}$ & $\begin{array}{c}\text { 电导率/ } \\
\mu \mathrm{S} \cdot \mathrm{cm}^{-1}\end{array}$ \\
\hline Somos14120 & 321.2 & 37.86 & 1.4 \\
丙三醇 & 1623.3 & 60.81 & 0.048 \\
二氧化钛溶胶 & 19.3 & 25.73 & 30 \\
液体石蜡 & 32.1 & 30.53 & 0.12 \\
\hline
\end{tabular}

Somos 14120、丙三醇、液体石蜡可直接作为流 体应用. 二氧化钛前驱体溶胶的制备方法如下: 先将 $1.0 \mathrm{~g} \mathrm{PVP}(\mathrm{K} 30)$ 粉末溶于 $2.0 \mathrm{~g}$ 无水乙醇与 $4.0 \mathrm{~g}$ 冰醋 酸中, 完全溶解后加入 $7.0 \mathrm{~g}$ 钛酸四正丁酯, 搅拌约 1 $\mathrm{h}$ 使其形成均一溶液. 普通染料的浓度约为 $1 \%$ (质量 浓度).

复合电喷实验装置如图 1 所示, 以两室微胶囊的 制备为例, 两根内管与外管组装形成复合喷头. 两内 管在外内管内独立分布, 操作时内管内分别通入两 种不同的核流体，外管内通入壳流体. 对于聚合物两 室微胶囊的制备, Somos 14120 以 $8.0 \mathrm{~mL} / \mathrm{h}$ 的速度从
外管流出, 染色的丙三醇以 $0.2 \mathrm{~mL} / \mathrm{h}$ 的速度分别从 两个内管流出. 工作电压约为 $20 \sim 30 \mathrm{kV}$, 工作距离为 $10 \mathrm{~cm}$. 对于有机/无机复合两室微胶囊的制备, 二氧 化钛前驱体溶胶以 $4.0 \mathrm{~mL} / \mathrm{h}$ 的速度通向外管, 液体 石蜡以 $0.2 \mathrm{~mL} / \mathrm{h}$ 的速度分别通向两个内管. 工作电 压约为 $25 \sim 35 \mathrm{kV}$, 工作距离为 $35 \mathrm{~cm}$. 复合喷头与高 压电源的正极相连, 接地金属板表面覆盖一层铝箔 作为接收装置. 对于显微镜表征, 产品直接收集在载 玻片上. 更多室结构微胶囊的制备需将相应数目的 内管组装于适当的外管并通入相应的流体进行复合 电喷.

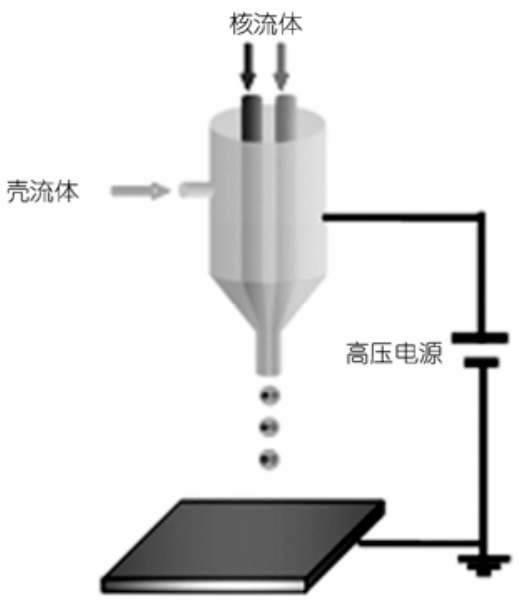

图 1 两室微胶囊制备装置示意图

JEOL FE-SEM 6700F 扫描电子显微镜用来表征 产品的表面形貌及断面. 对于断面表征, 产品首先经 过煅烧去除有机物质然后研碎展现断面. 升温速率 为 $1{ }^{\circ} \mathrm{C} / \mathrm{min}, 500^{\circ} \mathrm{C}$ 煅烧 $2 \mathrm{~h}$, 自然冷却. JEOL JEM-2010 透射电子显微镜用来表征产品的内部结 构. 为提高图像的对比度产品同样经过了如上述的 煅烧处理. 加速电压为 $200 \mathrm{kV}$. Leica DM4000M 显微 镜 (德国) 用来进行显微镜表征. 电化学工作站 CHI630B(上海)表征复合电喷过程中的电流电压性质. SNB-1 旋转黏度计(上海)表征流体黏度, DDS- II A 电 导率仪表征流体电导率, Dataphysics DCAT 11 (德国) 表征流体的表面张力. Spellman SL50P60 高压电源 (美国)提供直流高压以及 NE-1600 注射洜控制流体流 速. 照片由 Fujifilm 数码相机拍摄.

\section{2 结果与讨论}

我们对传统电喷装置的喷头系统进行了设计, 将 
一定数目的较细的内管插于较粗的外管组装了具有分 级结构的复合喷头. 操作时内管内分别通入不同的核 流体, 外管内通入壳流体. 采用如图 1 所示的装置我们 以光敏环氧树脂 Somos 14120 为壳流体通入到外管中, 分别染色的丙三醇为两种核流体通入到两内管中进行 复合电喷. 由于两内管在内管中不接触, Somos 由内管 之间以及内管与外管之间的空隙流出, 从而形成了对 丙三醇核流体的完全并且独立的包覆. 如图 2(a)所示, 由于核壳流体不混溶, 三者相遇后在喷口处形成边界 分明的复合液滴. 当施加足够大的电压, 该复合液滴首 先被共同拉伸形成雉(Taylor 锥), 而后在雉顶形成射流 (图 2(b)), 射流被拉伸一段距离后发生分裂形成带电液 滴喷雾. 将其收集用光学显微镜进行表征, 发现为两丙 三醇独立封装的双室结构微胶囊, 如图 2(c)所示. 壳层 聚合物可进一步用 $365 \mathrm{~nm}$ 紫外光照射将其固化.

从整个过程可以可出, 正是由于喷头的独特结 构保证了整个电喷过程中壳流体形成对核流体的有
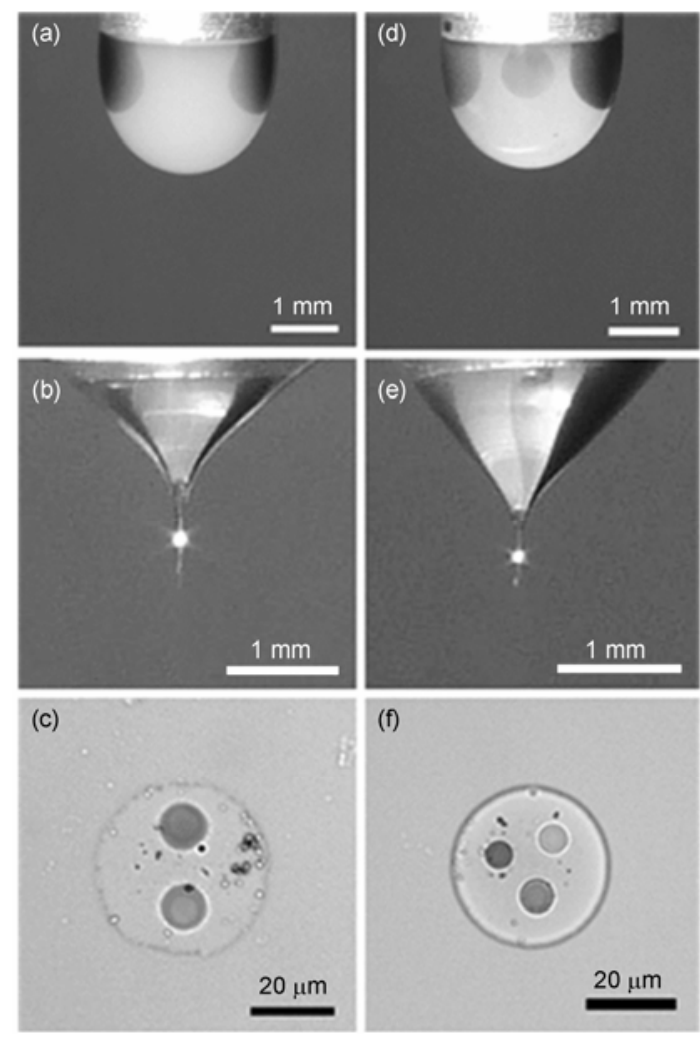

(f)

图 2

(a), (d)分别为两种、三种丙三醇核流体与 Somos 壳流体在喷口处 形成的复合液滴的数码照片; (b), (e)分别为二、三核流体体系形成 的 Taylor 雉照片; (c), (f) 分别为二、三核流体体系形成的液体微胶 囊显微镜照片
效并且独立的包覆，从而导致了多组分同时包覆的 多室结构微胶囊的形成 ${ }^{[4,22]}$.

微胶囊内部腔室的数目可通过设计复合喷头构 造来方便的控制. 复合喷头内管的数目与胶囊内部 腔体的数目是一致的, 操作时一定数目的内管通入 不同的核流体即对应于相应数目的内腔室及核组分 的封装. 例如, 一个内管对应于单室单组分的封装; 二、三、四个内管则分别对应于二、三、四种核组分 的独立封装. 我们进一步将上述体系中复合喷头中 内管的数目增至 3 个并保持他们在外管内部成正三 角形独立分布, 同时通入 3 种核流体(分别染成红、 黄、蓝色的丙三醇), 结果可获得 3 组分独立封装的 3 室微胶囊(图 2(d), (e), (f)).

同样采用如图 1 所示的装置我们以二氧化钛前驱 体溶胶为壳流体, 液体石蜡为两核流体进行复合电 喷制备二氧化钛/PVP复合微胶囊. 与液态光敏树脂 不同, 二氧化钛前驱体溶胶为溶液体系, 在电喷过程 中会发生溶胶-凝胶缩聚反应及溶剂挥发而固化, 因 而最后产品的尺寸较分裂生成的液滴的尺寸大大缩 小, 收集到的产品为一层白色的尺寸在微米至亚微 米之间的球形粒子(图 3(a)). 粒子的尺寸分布可通过 进一步调节实验参数来减小 ${ }^{[7.23-27]}$. 透射电子显微镜 照片(图 3(b))显示单个粒子内部具有双室结构, 由一 道连续的壁相隔, 类似于希腊字母“ $\boldsymbol{O}$ ”结构. 断面扫 描电子显微镜照片(图 3(c)) 进一步证实了双室结构的 存在, 虽然中间的隔壁很薄 (100 nm左右), 但是是完 整连续的. 这说明这种双室结构可以有效地分隔和 保护封装的两种核组分. 同样由于壳流体固化过程 导致的体积大幅收缩, 使壳材料对液态的核流体不 断进行压缩, 最终形成类似于“ $O$ ”的双室结构而不是 如上述光敏聚合物体系的圆形室结构. 我们进一步 将 3 个内管与外管组装形成多级复合喷头, 并相应的 通入液体石蜡核流体, 结果发现可获得具有 3 室的微 胶囊, 如图 3(d)所示. 产品结构与复合喷头结构具有 良好的一致性, 说明该方法具有简易性和良好的可 操作性. 在上述复合电喷过程中, 液体石蜡核流体是 不导电的, 因而不能单独进行电喷. 但是在适当的电 压下它能随着壳层的二氧化钛前驱体溶胶共同被拉 伸, 最后被包覆于壳材料中形成多室结构的产品. 这 是因为壳流体能够将作用于它与空气界面上的切向 电场力通过本身的秥度传送到体相直至它与核流 体的界面上. 因而在足够大的电场力作用下, 具有合 


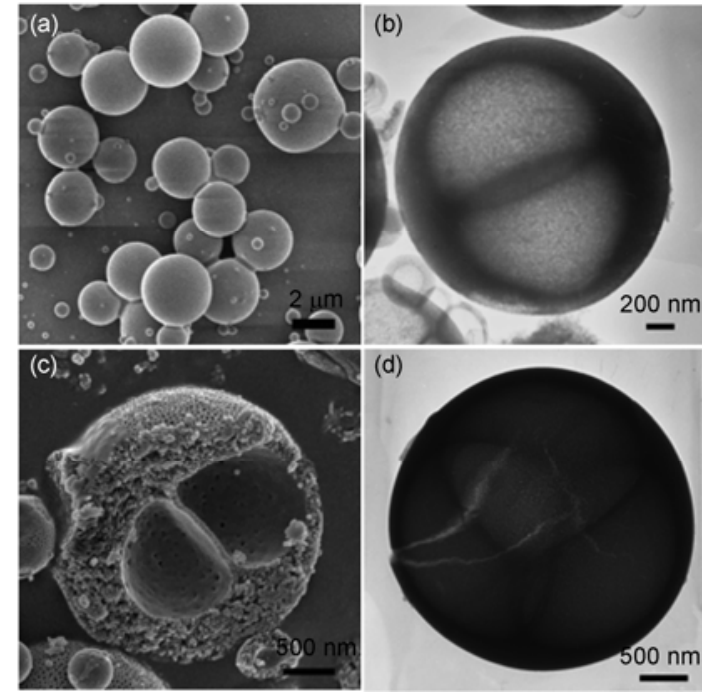

图 3

(a) 两室二氧化钛/PVP 复合微胶囊的扫描电子显微镜照片; (b) 两室二氧化钛/PVP复合微胶囊煅烧后的透射电子显微镜照片;

(c) 两室二氧化钛/PVP 复合微胶囊炍烧后的断面扫描电子显微镜照片;

(d) 三室二氧化钛/PVP 复合微胶囊烧烧后的透射电子显微镜照片

适黏度的壳流体可以驱动绝缘的内流体随其一起运 动形成复合射流, 经分裂、固化, 最后形成产品(如果 电场力太小或壳流体粘度太低, 可能不能形成稳定连 续的驱动作用). 我们测量了电喷过程中射流传输的 电流. 结果表明, 当固定核流体的流速而逐步增大壳 流体的流速, 射流传输的电流逐渐增大(图 4(a)); 当 固定壳流体的流速而逐渐增大核流体的流速, 射流 传输的电流变化不大(图 4(b)). 这充分说明复合电喷 过程中电荷分布在壳流体上, 电场力主要作用于导 电的壳流体上, 而核流体的运动则由壳流体驱动 ${ }^{[28]}$.

与目前报道的多室微胶囊制备方法相比, 我们 提出的这种多流体复合电喷技术具有很大优势: (1) 操作简单, 可一步实现多室结构的制备, 进而实现多 种组分的同时并且独立封装, 尤其适用于活性材料 的封装; (2) 装置比较简易; (3) 适用性广, 不同于自 组装法或微流体法受材料选择的限制比较大, 该法 可方便的扩展到很多其他功能材料的制备. 简易的 操作, 多种组分的同时封装, 独立腔室结构对核组分 的保护使其在生物医药领域具有很好的应用前景. 例如可在同一个胶囊内同时封装可特异性结合的抗
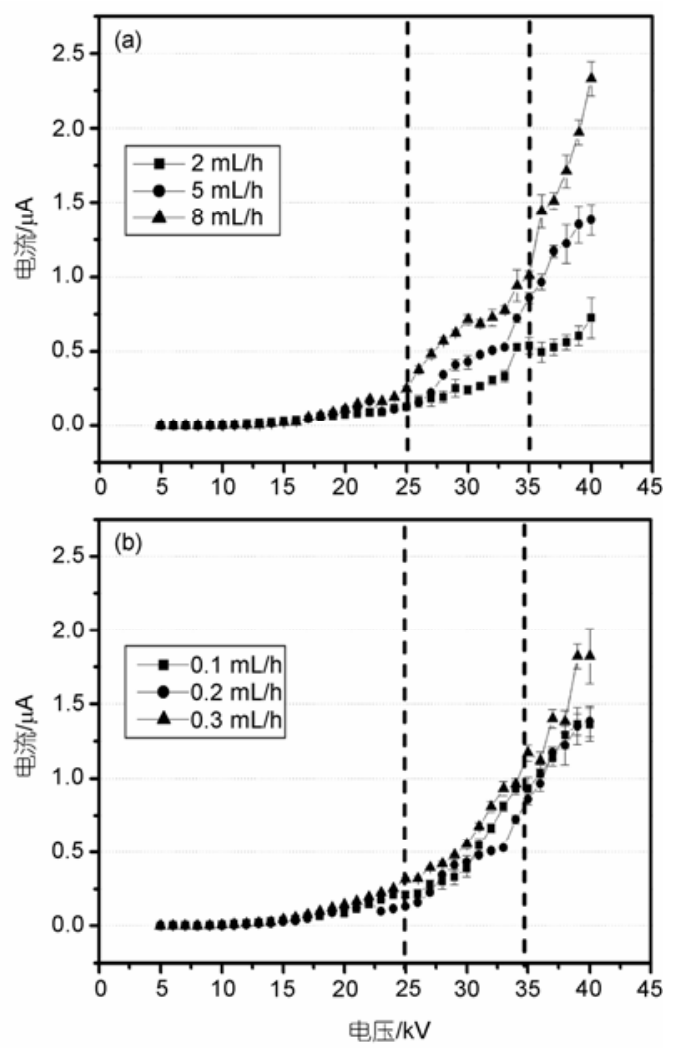

图 4

(a) 液体石蜡/二氧化钛溶胶体系复合电喷过程中随二氧化钛溶胶 壳流体流速变化复合射流的电流-电压曲线. 两液体石蜡核流体 的流速固定为 $0.2 \mathrm{~mL} / \mathrm{h}$; (b) 该体系随液体石蜡核流体流速变化 (两核流体流速保持相等, 共同变化)复合射流的电流-电压曲线. 二氧化钛溶胶壳流体的流速固定为 $5 \mathrm{~mL} / \mathrm{h}$. 虚线区间表示本体系 实验采用的工作区间

原和抗体, 多室结构的存在可以保证二者的稳定存在, 并且简单的操作步骤可以大大降低材料失活的几率. 另外由于方法的适用性广, 可以采用多种材料作为壳 组分, 从而赋予胶囊更丰富的功能(如靶向、缓释等).

\section{3 结论}

我们提出的多流体复合电喷技术可以通过一步 操作即可将多种组分封装到同一个胶囊当中, 并且同 时形成的多室结构对各封装的组分进行了分隔, 实现 了对各组分分布的精确控制以及保护, 尤其适用于活 性材料同时封装. 这种新颖的方法将在智能药物输 运、生物微反应器等领域具有潜在的应用前景. 
idic devices. Langmuir, 2004, 20: 9905-9908[DOD]

2 Peyratout C S, Dähne L. Tailor-made polyelectrolyte microcapsules: from multilayers to smart containers. Angew Chem Int Ed, 2004, 43: $3762-3783 \underline{[\mathrm{DOI}]}$

3 Utada A S, Lorenceau E, Link D R, et al. Monodisperse doubleemulsions generated from a microcapillary device. Science, 2005, 308: $537-541 \underline{[\mathrm{DOI}]}$

4 Loscertales I G, Barrero A, Guerrero I, et al. Micro/nano encapsulation via electrified coaxial liquid jets. Science, 2002, 295: 1695$1698 \underline{\mathrm{DOI}]}$

5 Larsen G, Velarde-Ortiz R, Minchow K, et al. A method for making inorganic and hybrid (organic/inorganic) fibers and vesicles with diameters in the submicrometer and micrometer range via sol-gel chemistry and electrically forced liquid jets. J Am Chem Soc, 2003, 125: $1154-1155$ [DOI]

6 Marín Á G, Loscertales I G, Márquez M, et al. Simple and double emulsions via coaxial jet electrosprays. Phys Rev Lett, 2007, 98: $014502 \underline{\text { DOI] }}$

7 Chen X, Jia L, Yin X, et al. Spraying modes in coaxial jet electrospray with outer driving liquid. J Phys Fluids, 2005, 17: 032101 [DOI]

8 Li D, Xia Y. Direct fabrication of composite and ceramic Hollow nanofibers by electrospinning. Nano Lett. 2004, 4: 933-938[DOI]

9 Sun Z, Zussman E, Yarin A L, et al. Compound core-shell polymer nanofibers by co-electrospinning. Adv Mater, 2003, 15: 1929$1932 \underline{\text { DOI] }}$

10 Yu J H, Fridrikh S V, Rutledge G C. Production of submicrometer diameter fibers by two-fluid electrospinning. Adv Mater, 2004, 16: $1562-1566 \underline{[\mathrm{DOI}]}$

11 Kreft O, Prevot M, Möhwald H, et al. Shell-in-shell microcapsules: A novel tool for integrated, spatially confined enzymatic reactions. Angew Chem Int Ed, 2007, 46: 5605-5608[DOI]

12 Kreft O, Skirtach A G, Sukhorukov G B, et al. Remote control of bioreactions in multicompartment capsules. Adv Mater, 2007, 19: $3142-3145 \underline{[\mathrm{DOI}]}$

13 Bolinger P -Y, Stamou D, Vogel H. Integrated nanoreactor systems: triggering the release and mixing of compounds inside single vesicles. J Am Chem Soc, 2004, 126: 8594-8595[DOI]

14 Gohy J-F, Willet N, Varshney S, et al. Core- shell - corona micelles with a responsive shell. Angew Chem Int Ed, 2001, 40: 3214$3216 \underline{\text { DOI] }}$

15 Zhou Z, Li Z, Ren Y, et al. Micellar shape change and internal segregation induced by chemical modification of a triptych block copolymer surfactant. J Am Chem Soc, 2003, 125: 10182-10183[DOI]

16 Li Z, Kesselman E, Talmon Y, et al. Multicompartment micelles from ABC miktoarm stars in water. Science, 2004, 306: 98$101 \underline{\text { DOI] }}$

17 Thünemann A F, Kubowicz S, Berlepsch H, et al. Two-compartment micellar assemblies obtained via aqueous self-organization of synthetic polymer building blocks. Langmuir, 2006, 22: 2506-2510[DOI]

18 Chu L Y, Utada A S, Shah R K, et al. Controllable monodisperse multiple emulsions. Angew Chem Int Ed, 2007, 46: 8970$8974 \underline{[\mathrm{DOI}]}$

19 Roh K H, Martin D C, Lahann J. Biphasic Janus particles with nanoscale anisotropy. Nat Mater, 2005, 4: 759-763[DOI]

20 Roh K H, Martin D C, Lahann J. Triphasic nanocolloids. J Am Chem Soc, 2006, 128: 6796-6797[DOI]

21 Chen H, Zhao Y, Song Y, et al. One-step multicomponent encapsulation by compound-fluidic electrospray. J Am Chem Soc, 2008, 130: $7800-7801$ [DOI]

22 Zhao Y, Cao X, Jiang L. Bio-mimic multichannel microtubes by a facile method. J Am Chem Soc, 2007, 129: 764-765[DOI]

23 Berkland C, Pack D W, Kim K. Controlling surface nano-structure using flow-limited field-injection electrostatic spraying (FFESS) of poly(d,1-lactide-co-glycolide). Biomaterials, 2004, 25: 5649-5658[DOI]

24 Xie J, Marijnissen J C M, Wang C-H. Microparticles developed by electrohydrodynamic atomization for the local delivery of anticancer drug to treat C6 glioma in vitro. Biomaterials, 2006, 27: 3321 - 3332[DOI]

25 Fantini D, Zanetti M, Costa L. Polystyrene microspheres and nanospheres produced by electrospray. Macromol Rapid Commun, 2006, 27: $2038-2042 \underline{[\mathrm{DOI}]}$

26 Xu Y, Hanna M A. Electrospray encapsulation of water-soluble protein with polylactide Effects of formulations on morphology, encapsulation efficiency and release profile of particles. Int J Pharm, 2006, 320: 30-36[DOI]

27 Jiang L, Zhao Y, Zhai J. A lotus-leaf-like superhydrophobic surface: a porous microsphere/nanofiber composite film prepared by electrohydrodynamics. Angew Chem Int Ed, 2004, 43: 4338 - 4341ㅁD]

28 López-Herreraa J M, Barreroa A, López A, et al. Coaxial jets generated from electrified Taylor cones. Scaling laws. J Aerosol Sci, 2003, 34: 535-552[DOI] 